\title{
PARABOLICS ON THE BOUNDARY OF THE DEFORMATION SPACE OF A KLEINIAN GROUP
}

\author{
JAMES W. ANDERSON
}

(Communicated by Ronald Stern)

\begin{abstract}
We present a condition on a loxodromic element $L$ of a Kleinian group $G$ which guarantees that $L$ cannot be made parabolic on the boundary of the deformation space of $G$, namely, that the fixed points of $L$ are separated by the limit set of a subgroup $F$ of $G$ which is a finitely generated quasifuchsian group of the first kind. The proof uses the collar theorem for short geodesics in hyperbolic 3-manifolds.
\end{abstract}

\section{INTRODUCTION}

In [3], Maskit shows that any loxodromic element $L$ in a function group $G$ which represents a simple loop on $\Omega(G) / G$ can be made parabolic on $\partial T(G)$; that is, there is a $\varphi \in \partial T(G)$ with $\varphi(L)$ parabolic. This was generalized by Ohshika [4] to all geometrically finite $G$.

We consider here the converse question of which loxodromic elements of $G$ can be made parabolic on the boundary of $T(G)$. For geometrically finite $G$, a complete answer to this question would give a 'combinatorial' description of all geometrically finite points on $\partial T(G)$.

Using the collar theorem of Brooks and Matelski [1] for short geodesics in hyperbolic 3-manifolds, we show that any loxodromic whose fixed points are separated by the limit set of a finitely generated quasifuchsian group of the first kind cannot be made parabolic on $\partial T(G)$.

\section{DEFINITIONS AND PRELIMINARIES}

We use [2] as our standard reference for definitions.

By a quasifuchsian group, we will mean a finitely generated quasifuchsian group of the first kind.

Given a Kleinian group $G$, a quasifuchsian subgroup $F$ of $G$, and a loxodromic $L \in G$, say that $L$ is separated by $F$ if the fixed points of $L$ are separated by $\Lambda(F)$. If $L$ is separated by $F$, then neither fixed point of $L$ lies in $\Lambda(F)$, and so $\langle L\rangle \cap F$ is trivial.

The deformation space $T(G)$ of a finitely generated Kleinian group is the set of discrete, faithful representations of $G$ into $\operatorname{PSL}_{2}(\mathbf{C})$ which are induced

Received by the editors April 25, 1993.

1991 Mathematics Subject Classification. Primary 57M50, 30F40.

Key words and phrases. Parabolic element, deformation space, collar theorem. 
by quasiconformal maps from $\overline{\mathbf{C}}$ to itself, modulo conjugation. That is, for $\varphi \in T(G)$, there is a quasiconformal map $f$ of $\overline{\mathbf{C}}$ to itself, so that $\varphi(g)=$ $f g f^{-1}$ for all $g \in G$. In particular, if $L$ is separated by $F$ in $G$, then $\varphi(L)$ is separated by $\varphi(F)$ in $\varphi(G)$ for all $\varphi \in T(G)$.

Given a (primitive) loxodromic $L$ in a Kleinian group $G$, a collar for $L$ in $G$ is a tubular neighborhood of the axis of $L$ in $\mathbf{H}^{3}$ which is precisely invariant under $\langle L\rangle$ in $G$.

The collar theorem of Brooks and Matelski gives an explicit formula for the radius of a collar for $L$ in terms of the multiplier of $L$, provided this multiplier is sufficiently close to 1 .

Theorem 2.1 [1]. Given $\tau$ such that $|\sinh (\tau / 2)|<1 / \sqrt{6}$, every element $L$ of a Kleinian group $G$ with $\operatorname{tr}(L)= \pm 2 \cosh (\tau / 2)$ has a collar of radius $r(\tau)$, where $r(\tau)$ is defined by

$$
\sinh (r(\tau))=\sqrt{\frac{1}{4|\sinh (\tau / 2)|^{2}}-\frac{3}{2}} .
$$

In particular, there is an absolute constant $\varepsilon_{0}>0$ and a function $d:\left(0, \varepsilon_{0}\right)$ $\rightarrow \mathbf{R}$, so that, for any Kleinian group $G$ and any loxodromic $L \in G$ with $\left|\operatorname{tr}^{2}(L)-4\right| \leq 4 \varepsilon<4 \varepsilon_{0}$, there is a collar of radius $d(\varepsilon)$ for $L$ in $G$. Moreover, $d(\varepsilon) \rightarrow \infty$ as $\varepsilon \rightarrow 0$.

\section{Results}

We are ready to prove the main result of this note.

Theorem 3.1. Let $G$ be a finitely generated Kleinian group, and let $F$ be a quasifuchsian subgroup. There exists $c>0$, dependent on $F$ but independent of $G$, so that $\left|\operatorname{tr}^{2}(\varphi(L))-4\right| \geq c$ for all $\varphi \in T(G)$ and all loxodromics $L \in G$ separated by $F$.

Proof. Assume that there exist $\varphi_{k} \in T(G)$ so that $\left|\operatorname{tr}^{2}\left(\varphi_{k}(L)\right)-4\right| \rightarrow 0$ as $k \rightarrow \infty$.

Let $H_{k}$ be the convex hull of $\Lambda\left(\varphi_{k}(F)\right)$ in $\mathbf{H}^{3}$. Since the $\varphi_{k}(F)$ are isomorphic for all $k$, the area of the pleated hyperbolic surface $S_{k}=\partial H_{k} / \varphi_{k}(F)$ is constant.

Using Theorem 2.1, we can find $\varepsilon_{k}$, going to $\infty$ as $k \rightarrow \infty$, so that $\varphi_{k}(L)$ has a collar of radius $\varepsilon_{k}$ in $\varphi_{k}(G)$.

Let $x_{k}$ be a point of intersection of the axis of $\varphi_{k}(L)$ with the boundary of $H_{k}$. Since $\left\langle\varphi_{k}(L)\right\rangle \cap \varphi_{k}(F)$ is trivial, the ball $B_{k}$ of radius $\varepsilon_{k}$ about $x_{k}$ is precisely invariant under the identity in $\varphi_{k}(F)$.

The intersection of $B_{k}$ with $\partial H_{k}$ contains a disc $D_{k}$ of radius $\varepsilon_{k}$ which contains $x_{k}$. Since $D_{k}$ is precisely invariant under the identity in $\varphi_{k}(F)$, it projects to a disc of radius $\varepsilon_{k}$ on $S_{k}$.

As $k \rightarrow \infty$, the areas of the $D_{k}$ go to infinity, and so the areas of the $S_{k}$ go to $\infty$. This gives the desired contradiction.

Corollary 3.2. Let $G$ be a finitely generated Kleinian group, and let $L \in G$ be a loxodromic separated by a quasifuchsian subgroup of $G$. Then $\varphi(L)$ is loxodromic for all $\varphi \in \partial T(G)$.

Quasifuchsian subgroups of Kleinian groups give rise to (not necessarily embedded) incompressible surfaces in the corresponding 3-manifolds. In some 
sense, this theorem gives a collar theorem about these surfaces, namely, that the complex length of any closed geodesic crossing this surface is bounded from below.

\section{REFERENCES}

1. R. Brooks and J. Matelski, Collars in Kleinian groups, Duke Math. J. 49 (1982), 163-182.

2. B. Maskit, Kleinian groups, Springer-Verlag, New York, 1988.

3. __ Parabolic elements in Kleinian groups, Ann. of Math. (2) 117 (1983), 659-668.

4. K. Ohshika, Geometrically finite Kleinian groups and parabolic elements, preprint.

Department of Mathematics, Rice University, P. O. BoX 1892, Houston, Texas 77251 E-mail address: jwa@math.rice.edu 